\title{
Introduction: economics with memory
}

The birth of modern economics occurred almost simultaneously with the appearance of new economic concepts, which began to be actively used in various economic models. "Marginal revolution" and "Keynesian revolution" in economics introduced fundamental economic concepts, including the concepts of "marginal value," "economic multiplier," "economic accelerator," "elasticity" and many others. These concepts are based on the use of mathematical tools, which were not previously used in the economics. The most important mathematical tools, which are become actively used in description of economic processes, are the theory of derivatives and integrals of integer orders, the theory of differential and difference equations. These mathematical tools allowed economists to build economic models in a mathematical form and on their basis to describe a wide range of economic processes and phenomena. "Marginal revolution" and "Keynesian revolution" led to the use of standard mathematical calculus, which is theory of the derivatives and integrals of integer orders, and the differential and difference equations of integer orders.

As a result of the past marginal and Keynesian revolutions, the economic models with continuous and discrete time began to be described by differential equations with derivatives of integer orders and equations with finite differences of integer orders. However, these tools have a number of shortcomings that lead to the incompleteness of descriptions of economic processes. It is known that the derivatives of integer orders are determined by the properties of a differentiable function only in an infinitely small neighborhood of the point, in which these derivatives are considered. As a result, differential equations with derivatives of integer orders, which are used in economic models, cannot describe processes with memory and nonlocality in time. In fact, such equations describe only economic processes, in which all economic agents have complete amnesia and they do not remember the past events in the economy. Obviously, this assumption about the absence of memory among economic agents is a strong restriction for economic models. Therefore, standard models have drawbacks, since they cannot take into account the important aspects of economic processes and phenomena.

At the present moment, a new revolution, which can be called "Memory revolution," is actually taking place in modern economics. This revolution is intended to "cure amnesia" of modern economic theory, which is caused by the use of differential and integral operators of integer orders. In economics, it becomes obvious that when describing the behavior of economic agents, we must take into account that agents may have memory. The description of economic processes should take into account that the behavior of economic agents may depend on the history of previous changes in economy. To describe this type of behavior, we need new economic concepts and notions that allow us to take into account the presence of memory in economic processes. New economic models and methods are needed to take into account that economic 
agents may remember the changes of economic indicators and factors in the past. The presence of memory changes the behavior of agents and influences the decisions they make. As a result, to describe economic processes with memory, we cannot use the standard tools of differential (or difference) equations of integer orders. In fact, these equations describe only such economic processes, in which agents actually have amnesia. In other words, economic models, which use only derivatives of integer orders, can be applied when economic agents forget the history of changes of economic indicators and factors during an infinitesimally small period of time. It is becoming clear that this restriction holds back the development of economic sciences.

The "Memory revolution" is intended to include in the modern economic theory new economic concepts and notions that allow us to take into account the presence of memory in economic processes. The main mathematical tool designed to "cure amnesia" in economics is the theory of derivatives and integrals of noninteger order (the fractional calculus), fractional differential and difference equations [335, 202, 308, $200,90,164,165$, 119]. Fractional calculus as a theory of integrals, derivatives, and finite differences of arbitrary (integer and noninteger) orders includes standard calculus as a special case. Derivatives and integrals of arbitrary (integer and noninteger) orders are well known in mathematics more than 300 . There are different types of fractional integral and differential operators that have been proposed by such well-known mathematicians as Riemann, Liouville, Grunwald, Letnikov, Sonine, Marchaud, Weyl, Riesz, Kober, Erdelyi and other scientists. In modern economics, fractional calculus is intended to replace standard calculus of integrals, derivatives and differences of integer orders.

The "Memory revolution" has led to the emergence of a new economic science, which can be called "Economics with Memory." The mathematical language of this science is fractional calculus, which allows us to formulate new concepts and principles. However, this does not mean that the economics with memory can be considered as part of applied mathematics or mathematical economics. Economics with memory is the science of the behavior of the behavior and interactions of economic agents with memory and economic processes with memory and nonlocality in time. Economics with Memory should be based on new economic concepts, notions, principles and models that allow us to describe economic processes with memory. The modern revolutionary situation in economic theory means that now an important task is the formulation of new economic concepts, concepts, models and principles. This is especially important due to the fact that the economics with memory is now only being formed as new branch of science.

The beginning of the "Memory revolution" in economics may be associated with the works, which were published in 1966 and 1980 by Clive W. J. Granger [147, 148, $152,155,153,151]$. Note that Granger received the Nobel memorial prize in economic sciences in 2003 "for methods of analyzing economic time series with common trends (cointegration)" [479]. For the first time, the importance of long-range time dependence in economic data was recognized by Clive W.J. Granger in his article [148] in 
1966 (see also [147, 152, 155, 29]). Granger showed that a number of spectral densities, which are estimated from economic time series, have a similar form. We can state that the phenomenon of long memory in modern economics was discovered by Granger. Then, to describe economic processes with memory Granger and Joyeux [155] in 1980 proposed the fractional ARIMA models, which are also called ARFIMA(p, d, q). The fractional ARIMA(p, d, q) models are generalization of $\operatorname{ARIMA}(p, d, q)$ model from a positive integer order $d$ to noninteger (positive and negative) orders $d[29,30]$. To generalize ARIMA models, Granger and Joyeux [155] (see also [153, 180]) proposed the so-called fractional differencing and integrating for discrete time case (see books $[29,295,30,481,231,485]$, and reviews [151, 21, 303, 23, 135]). The fractional difference operators of Granger and Joyeux were proposed and then began to be used in economics up to the present time without any connection with the fractional calculus and the well-known fractional differences of noninteger orders. In fact, these fractional differencing and integrating are the well-known Grunwald-Letnikov fractional differences, which were suggested in 1867 and 1868 in works [157, 222]. Then the GrunwaldLetnikov fractional differences are actively used in the fractional calculus [335, 308, 200] and began to apply in physics and other sciences. We should also note that in the continuous limit the Grunwald-Letnikov fractional differences of positive orders can give the Grunwald-Letnikov, Marchaud and Liouville fractional derivatives [335].

Approach, which is based on the discrete operators proposed by Granger and Joyeux is the most common among economists [21, 303, 23, 135]. The approach based on discrete operators of Granger-Joyeux, more precisely on the Grunwald-Letnikov fractional differences, is restricted by only one type of fractional finite differences. Unfortunately, this approach is used without an explicit connection with the modern mathematics and the development of fractional calculus in the last 200 years. It should be emphasized that the Granger-Joyeux approach to economics with memory is restricted by models with discrete time and application of the Grunwald-Letnikov fractional differences. Obviously, the restriction of mathematical tools to only to the discrete Grunwald-Letnikov operators significantly reduces the possibilities to describe processes with memory and nonlocality in time. In recent years, attempts have been made to suggest a different approach to the description of processes with memory in economics and finance. A brief description of the history of attempts to describe economic and finance processes with memory is given in work [385] (see also book [254, pp. 5-32]).

We can state that the use of fractional calculus in economic models will significantly expand the possibilities of describing processes with memory and will lead to new results. We propose to use all methods of modern fractional calculus to describe economic processes with memory, and to generalize the basic economic notions, concepts, models and principles to form a new economic science, the Economics with Memory. 
In this book, fractional calculus is used to formulate the concept of memory itself for economic processes, and to define basic concepts, models and principles for description of economic processes with memory and nonlocality in time.

From our subjective point of view, the formation of new basic economic concepts and notions of new science "Economics with Memory" began with a proposal of generalizations of the basic economic concepts and notions at the beginning of 2016, when the concept of elasticity for economic processes with memory was proposed in works [418] and [430, 419]. Then, in 2016, we proposed such new concepts as the marginal values with memory [424, 416, 425], accelerator with memory and multiplier with memory [412, 417], the nonlocal measures of risk aversion [428] and nonlocal deterministic factor analysis [414]. In 2016, these concepts are used in generalizations of some standard economic models [420, 421, 426, 423, 427, 422] that describe the dynamics of economic processes with memory.

During 2016-2020, we use the fractional calculus to define basic concepts of Economics with Memory, and to describe the dynamics of economic processes with memory. Some of these generalized economic concepts and models for processes with memory are described in the proposed book on economics with memory.

It should be noted that formal replacements of derivatives of integer order by fractional derivatives in standard differential equations, which describe economic processes, and solutions of the obtained fractional differential equations were considered in papers published before 2016. However, these papers were purely mathematical works, in which generalizations of economic concepts and notions were not proposed. In these works, fractional differential equations were not usually derived, since a formal replacement of integer-order derivatives by fractional derivatives cannot be recognized as a derivation of the equations. Formulations of economic conclusions and interpretations from the obtained solutions are not usually suggested in these papers. Examples of incorrectness and errors in such generalizations are given in [386], (see also book [254, pp. 43-92]). Review article [386] formulates five principles of the "fractional-dynamic" generalization of standard dynamic models. We can state that in the works with formal fractional generalizations of standard economic equations, the Principles of Derivability and Interpretability were neglected [386]. Let us give a brief formulation of the Principles of Derivability and Interpretability.

Derivability Principle: It is not enough to generalize the differential equations describing the dynamic model. It is necessary to generalize the whole scheme of obtaining (all steps of derivation) these equations from the basic principles, concepts and assumptions. In this sequential derivation of the equations, we should take into account the nonstandard characteristic properties of fractional derivatives and integrals. If necessary, generalizations of the notions, concepts and methods, which are used in this derivation, should also be obtained.

Interpretability Principle: The subject (physical, economic) interpretation of the mathematical results, including solutions and their properties, should be obtained. 
Differences and, first of all qualitative differences from the results obtained for the standard model, should be described.

Note that the problems and difficulties arising in the construction of generalized dynamic models with memory for the standard economic models by using the fractional calculus are described in [386] with details. The most important purpose of the "Memory revolution" is the inclusion of memory and time nonlocality into the economic theory, into the basic economic concepts and methods. The economics should be extended and generalized such that it takes into account the memory and time nonlocality. Generalizations of standard economic models should be constructed only on this conceptual basis. The most important purpose of studying such generalizations is the search and formulation of qualitatively new effects and phenomena caused by memory in the behavior of economic processes.

Let us list some generalizations of economic concepts and generalizations of economic models that have already been proposed in recent years.

The generalizations of some basic economic notions are described in the proposed book on Economics with memory. The list of these new notions and concepts primarily include the following:

1. the marginal value of noninteger order $[424,416,425,439,446]$ with memory and nonlocality;

2. the economic multiplier with memory [412, 397];

3. the economic accelerator with memory [412, 397];

4. the exact discretization of economic accelerators and multipliers [441, 442, 431, 432] based on exact fractional differences;

5. the accelerator with memory and periodic sharp bursts [417, 436, 444];

6. the duality of the multiplier with memory and the accelerator with memory [412, 397];

7. the accelerators and multipliers with memory and distributed lag [405, 404, 402];

8. the elasticity of fractional order [418, 430, 429, 419] for processes with memory and nonlocality;

9. the measures of risk aversion with nonlocality and with memory [428, 449];

10. the warranted (technological) rate of growth with memory $[450,447,445,453,393$, 387];

11. the nonlocal methods of deterministic factor analysis [414, 452];

12. the productivity with fatigue and memory [454];

13. the chronological memory ordering [433, 398];

14. and some other.

The use of these notions and concepts makes it possible for us to generalize some classical economic models, which are proposed by the following well-known economists:

1. Henry Roy F. Harrod [170, 171, 172] and Evsey D. Domar [92, 93];

2. John M. Keynes [191, 193, 194, 192];

3. Wassily W. Leontief [219, 220, 354, 355]; 


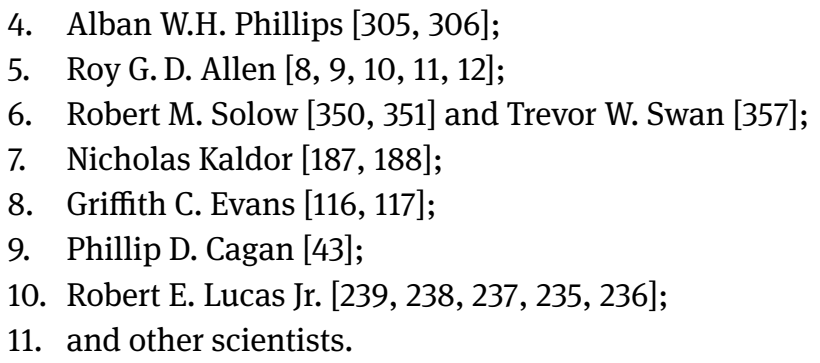

Economic models with continuous time are usually described by differential equations with derivatives of integer orders. In the standard economic models, the memory effects and memory fading are neglected. At the same time, it is obvious that it necessary to take into account the influence of memory effects on economic processes. We should take into account the memory, since the assumption of a lack of memory among economic agents, that is, the assumption that economic agents have lost their memory and have amnesia is a very strong restriction on economic models.

The presence of memory in the economic process means that the behavior of the process depends not only on the variables and parameters of this process at the present time, but also on the history of changes in these variables and parameters on a finite time interval. The concept of memory is very important for an adequate description of real economic processes.

From a mathematical point of view, the neglect of memory effects in standard economic models is due to the used equations with derivatives of integer orders, which are determined by the properties of the function in an infinitely small neighborhood of the considered time.

A very effective and powerful tool for describing memory effects is fractional calculus of derivatives and integrals of noninteger orders [335, 202, 308, 200, 90, 164, 165], which have a long history of more than 300 years [221, 328, 330, 329, 203, 467], $[469,470,489,464]$. We should note that the characteristic property of fractional derivatives of noninteger order is the violations of standard rules and properties that are fulfilled for derivatives of integer order [367, 287, 377, 376, 382]. These nonstandard mathematical properties allow us to describe nonstandard processes and phenomena associated with nonlocality and memory [386, 406, 385]. On the other hand, these nonstandard properties lead to difficulties [386] in sequential constructing generalizations of standard models. In article [386] (see also book [254, pp. 43-92]), we show how problems arise when building generalizations of standard dynamic models of economics.

The need to take into account the non-standard properties of fractional derivatives and integrals leads us to the following [386]:

First of all, it is not enough to generalize the differential equations describing the standard dynamic model. It is necessary to generalize the whole scheme of obtaining (all steps of derivation) these equations from the basic principles, concepts and 
assumptions (see the Derivability Principle in [386]). Therefore, it is necessary to consider the possibility of generalizing all stages of obtaining equations of standard economic models.

Second, in this sequential derivation of the equations, we should take into account the nonstandard characteristic properties of fractional derivatives and integrals. This leads us to the fact that it is necessary to take into account the violation of standard product and chain rules, the violation of semigroup properties for the repeated action of fractional and integer derivatives.

Third, for standard models, there is a set of various generalizations, due to the existence of various types of fractional operators and violation of s-equivalence for fractional differential equations (see the Multiplicity Principle in [386]).

Because of this, in this book, we restrict ourselves mainly to such generalizations of standard models in which there are exact analytical solutions of models equations.

In this book, we propose some economic models, in which effects of fading memory are taken into account. We describe the possible difficulties and ambiguities that arise in the generalization of standard economic models. We derive the fractional differential equations of the proposed economic models with memory and, then we find solutions of these equations. Using these solutions, we formulate some principles of economic dynamics with fading memory. For generalized models with memory, we describe differences from standard models that arise due to the presence of memory in the processes.

In this book, we will describe various economic models with fading memory, which are generalizations of the classical models. For example, the following economic models were proposed.

1. the natural growth model with memory [420, 440];

2. the growth model with constant pace and memory [447, 438];

3. the Harrod-Domar model with memory [421, 426] and [450, 445, 453, 393];

4. the Keynes model with memory [423, 427, 422] and [402, 407];

5. the dynamic Leontief (intersectoral) model with memory [437, 451, 433, 398];

6. the model of dynamics of fixed assets (or capital stock) with memory [447, 438];

7. the model of price dynamics with memory [438];

8. the logistic growth model with memory [386] (see also [403]);

9. the model of logistic growth with memory and periodic sharp splashes (kicks) [444];

10. the time-dependent dynamic intersectoral model with memory [433, 398];

11. the Phillips model with memory and distributed lag [405];

12. the Harrod-Domar growth model with memory and distributed lag [404];

13. the dynamic Keynesian model with memory and distributed lag [402, 407];

14. the model of productivity with fatigue and memory [454];

15. the Solow-Swan model with memory [386, 383];

16. the Kaldor-type model of business cycles with memory [386];

17. the Evans model with memory [390]; 
18. the Cagan model with memory [388];

19. the Lucas models with memory [391, 389, 381];

20. and some other economic models.

We should note that the standard concepts and models, which do not take into account the fading memory, can be considered as special cases of the proposed generalized concepts and models with memory, when the parameter of memory fading takes integer values. For example, fractional differential equations of economic models with memory include differential equations of integer orders that describe standard models. The situation is similar for generalized economic concepts. For example, marginal value with power-law memory contains the standard marginal and average values as special cases corresponding to the fading parameter equal to zero and one, respectively.

In this book, we proposed new concepts, models and principles for Economics with Memory. Qualitatively new effects due to the presence of memory in the economic process are described. The most important element in the construction of the Economics with memory as a branch of economics are the formation of new notions, concepts, effects, phenomena, principles and methods, which are specific only to this branch.

In writing this book, an ambitious task was set to create and describe the foundation of the Economics with Memory as a new branch of the economics. For this purpose, generalizations of basic economic concepts, models and principles of economic theory were proposed. It is obvious that such an ambitious task cannot be completely solved within the framework of one book, despite its volume. Many questions, concepts, notions, models, methods and even sections of economics remained beyond the scope of this book. However, the authors hope that the proposed book will lead to new publications of articles and books on Economics with Memory, which will fill in the missing elements. In this case, we will consider that the proposed book has achieved its purpose. We hope that the "Economy with Memory" will become in the near future an independent and actively developing new branch of economics. 